\title{
Clinical Evaluation of Treatment of Gingival Recessions supported by Bayesian Statistics
}

\author{
Maria Dobreva $^{1}$, Veska Noncheva ${ }^{2}$, Ivan Chenchev $^{3}$
}

\begin{abstract}
The purpose of this study is to investigate treatment results of gingival recessions using coronally advanced flap (CAF) and platelet rich fibrin membrane (PRFm) with CAF and connective tissue graft (CTG). The reported work gets answers to the following open questions: Is the treatment of mucogingival defects a predictable procedure? Is the gender a factor affecting the outcome of coronally advanced flap root coverage procedure? To achieve this goal the authors followed the Creeping Attachment clinical parameter six months postoperatively.
\end{abstract}

\section{INTRODUCTION}

Gingival recession is a common manifestation in most populations. Gingival recession may be a concern for patients for a number of reasons such as root hypersensitivity, erosion, root caries, and aesthetics. Multiple gingival recessions may be a concern for patients with a high lip smile line. Studies on this surgical challenge mostly concern the treatment of recession defects. Multiple adjacent recession-type defects present a further challenge because several recessions must be treated at a single surgical session to minimize patient discomfort.

Gingival recession affects a significant proportion of the adult population including those with a good standard of oral hygiene. In addition to its unfavorable effect on aesthetics and self-esteem, gingival recession also is associated with destructive periodontal diseases and root caries.

2010 Mathematics Subject Classification. Primary: Obligatory! Secondary: Optional. Key words and phrases. Biostatistics, split mouth randomized controlled study, ANOVA-like model, Bayesian analysis, heterogeneous-variance model, two-way interactions. 
The prevalence, extent, and severity of gingival recession increased with age. Males in the U.S. adult population had higher prevalence and extent of gingival recession compared to females [1].

Clinical evaluations of the treatment of isolated and adjacent multiple gingival recessions based on both a Coronally Advanced Flap (CAF) alone or connective tissue graft (CTG) and in combination with a Platelet-Rich Fibrin $(\mathrm{CAF}+\mathrm{PRF})$ membrane are presented in [2]-[6]. The aim of these studies was to determine whether the addition of an autologous platelet rich fibrin (PRF) membrane to a coronally advanced flap (CAF) would improve the clinical outcome in terms of root coverage in the treatment of isolated and adjacent multiple gingival recession. Systemically healthy subjects each with single Miller's class I or II buccal recession defect were randomly assigned to control $(\mathrm{CAF}+\mathrm{CTG})$ or test $(\mathrm{CAF}+\mathrm{PRF})$ group. All patients who had been included in the study have received the treatment and turned up regularly for re-evaluation. Mean and standard deviation for the clinical variables have been calculated for each treatment.

The aim of the study described in [5], [7] was to determine whether the addition of an autologous platelet rich fibrin (PRF) membrane to a coronally advanced flap (CAF) would improve the clinical outcome in terms of root coverage in the treatment of isolated gingival recession. The result of this split mouth randomized controlled study evaluating the adjunctive effect of platelet rich fibrin to coronally advanced flap in Miller's class I or II recession defects is described in [4]. The statistical analysis was performed using frequent statistics with R software. The significance of the difference within and between groups before and after treatment was evaluated with the paired t test. Differences were considered statistically significant at level of significance 0.05 .

\section{Creeping Attachment Phenomenon}

Root coverage is a desired outcome of treatment. The root coverage may result from a mechanism known as creeping attachment, which is the postoperative migration of the gingival marginal tissue in a coronal direction over portions of a previously denuded root. This phenomenon can be detected up to 4 years after graft surgery.

Gingival grafting is a well-established pure mucogingival procedure for increasing the width of attached gingiva. Since its introduction in 1963, the procedure has proven reliable in increasing attached gingiva and stopping progressive gingival recession. However, only a few cases of creeping attachment after gingival grafting have been reported in the dental literature. 
Matter and Cimasoni described 5 factors that seemed to have a definite influence on creeping attachment: width of the recession, position of the graft, interproximal bone resorption, position of the tooth and the patient's dental hygiene [10]. Creeping attachment typically occurs within one to twelve months after the graft surgery. The amount of creeping attachment is unpredictable. In order to elucidate the mechanism of creeping attachment we try identifying some factors that could play a significant role in this interesting clinical finding.

This research involved 30 people at the age of 23 to 70 years with a total of 118 symmetrical Miller's Class I and Class II gingival recessions on different places of the jaws. All of the patients' gingival recessions were treated surgically. The recessions on one side of the jaw were treated with coronally advanced flap (CAF) combined with platelet rich fibrin membrane (test group), while the other side was treated with CAF combined with connective tissue graft (control group). The success of the operation was evaluated through the measurement of creeping attachment (CA). A 6-month postoperative measurement period is sufficient to evaluate the stability of the gingival margin.

The aim of our ongoing research is to get answers to the following questions: Is the treatment of mucogingival defects a predictable procedure? Is the gender a factor affecting the outcome of coronally advanced flap root coverage procedure?

\section{MODEL}

We want to predict CA values from two factors: method of surgery (type of graft) and of gender patient. Both factors have two levels, so there are four groups altogether denoted as Control M, Test M, Control F, Test F.

Each group's data is described as random variation around a central tendency. The central tendencies of the groups are conceptualized as deflections from the overall baseline.

Bayesian models are appropriate to such data structure, without having to make assumptions for homogeneity of variance across groups and normally distributed noise.

We will apply heavy-tailed distributions to accommodate outliers, along with hierarchical structure to accommodate heterogeneous variances in the different groups. In the context of the generalized linear model (GLM) this situation involves a linear function of two nominal predictors.

An important concept of models with multiple nominal predictors is interaction. Interaction means that the effect of a predictor depends on the level 
of another predictor. Interaction is the nonadditive influence of the factors. The average effects of the factors are called the main effects.

\section{HIERARCHICAL DEPENDENCY STRUCTURE}

Hierarchical diagram for the model is shown on Figure 1. We have data structure that consist of a metric predicted variable $\mathrm{CA}$ and two nominal predictors, gender and method of treatment. The datum is assumed to be student $\mathrm{t}$ distributed around the predicted value. The predicted value is the baseline plus deflections. All the parameters are given meaningfully structured noncommittal prior distributions. The within-group standard deviations are given broad uniform prior distributions. The baseline parameter, $\beta_{0}$, is given a normal prior distribution, made broad on the scale of the data. The group deflection parameters, $\beta_{j}$, are given a normal prior distribution that has a mean of zero, because the deflection parameters are supposed to sum to zero. Each group deflection is estimated separately from the other groups.

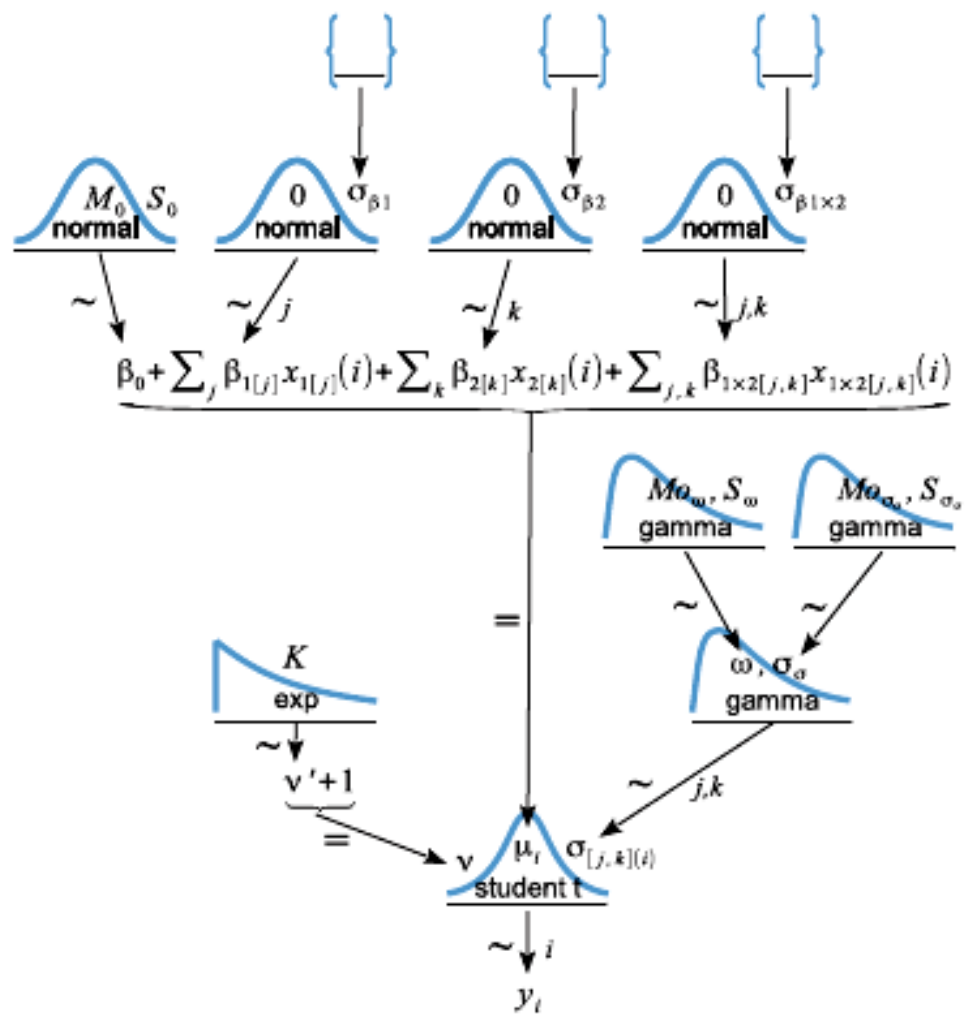

Figure 1: Hierarchical diagram for model that describes data from two nominal predictors. 
Each cell has its own $\sigma[j, k]$ parameter, and those parameters are described as being gamma-distributed across cells.

In our model $\sigma_{\beta 1 \times 2}$ is not a constant, which implies that the interaction deflections are mutually informative.

The goal in the Hierarchical Bayesian approach is to estimate the main and interaction deflections, and other parameters, based on the observed data [8], [9].

The goal of our analysis is to describe CA as a function of two nominal predictors: gender and method of treatment. There might be interactions, in the sense that the effect of CA might be of different magnitudes in male and female patients.

The basic results of the Bayesian data analysis are shown as the posterior predictive distributions superimposed on the data in Figure 2. The posterior distribution reveal joint probabilities of combinations of parameter values.
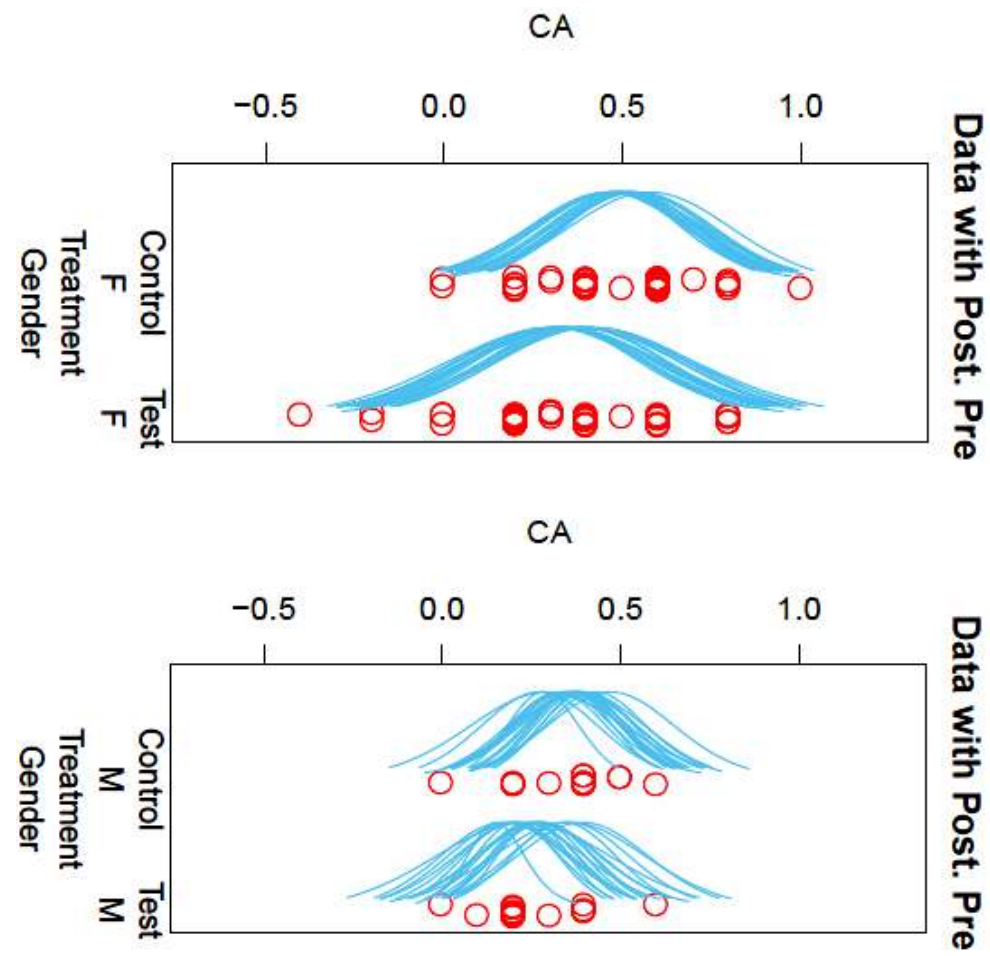

Figure 2: The posterior predictive distributions superimposed on the data.

Diagnostic graphics of chains for all the parameters are produced. The chains are converged at the sufficient length. 
In the Hierarchical Bayesian approach the emphasis is on estimation of the magnitudes of effects and their uncertainties.

For each parameter, Table 1 shows the estimated mean, median, mode, and 95\% HDI limits.

Table 1: Estimated parameters of the model in Figure1

\begin{tabular}{|l|c|c|c|c|c|c|}
\hline & Mean & Median & Mode & ESS & $\begin{array}{c}\text { 95\% } \\
\text { HDIlow }\end{array}$ & $\begin{array}{c}\text { 95\% } \\
\text { HDIhigh }\end{array}$ \\
\hline b0 & 0.36459 & 0.36443 & 0.36534 & 15717.70 & 0.32337 & 0.40746 \\
\hline b1[1] Control & 0.06771 & 0.06781 & 0.06723 & 12444.00 & 0.02586 & 0.10889 \\
\hline b1[2] Test & -0.06771 & -0.06781 & -0.06723 & 12444.00 & -0.10889 & -0.02586 \\
\hline b2[1] M & -0.05813 & -0.05829 & -0.06015 & 14207.00 & -0.09833 & -0.01506 \\
\hline b2[2] F & 0.05813 & 0.05829 & 0.06015 & 14207.00 & 0.01506 & 0.09833 \\
\hline b1b2[1,1] Control M & -0.01429 & -0.01280 & -0.00212 & 8833.90 & -0.05301 & 0.02034 \\
\hline b1b2[2,1] Test M & 0.01429 & 0.01280 & 0.00212 & 8833.90 & -0.02034 & 0.05301 \\
\hline b1b2[1,2] Control F & 0.01429 & 0.01280 & 0.00212 & 8833.90 & -0.02034 & 0.05301 \\
\hline b1b2[2,2] Test F & -0.01429 & -0.01280 & -0.00212 & 8833.90 & -0.05301 & 0.02034 \\
\hline m[1,1] Control M & 0.35988 & 0.35991 & 0.36008 & 11463.10 & 0.27185 & 0.45109 \\
\hline m[2,1] Test M & 0.25303 & 0.25216 & 0.24711 & 12726.20 & 0.16554 & 0.34151 \\
\hline m[1,2] Control F & 0.50471 & 0.50517 & 0.50916 & 13688.60 & 0.43999 & 0.56706 \\
\hline m[2,2] Test F & 0.34072 & 0.34089 & 0.34225 & 14459.70 & 0.25872 & 0.42166 \\
\hline a1SD & 0.32070 & 0.23289 & 0.10153 & 560.80 & 0.00069 & 0.91215 \\
\hline a2SD & 0.29145 & 0.20709 & 0.08440 & 566.40 & 0.00040 & 0.84576 \\
\hline a1a2SD & 0.14942 & 0.10646 & 0.04602 & 802.20 & 0.00004 & 0.42927 \\
\hline ySigma[1,1] Control M & 0.16795 & 0.16336 & 0.14959 & 14415.10 & 0.10397 & 0.24354 \\
\hline ySigma[2,1] Test M & 0.16288 & 0.15846 & 0.14965 & 14244.90 & 0.10029 & 0.23689 \\
\hline ySigma[1,2] Control F & 0.21059 & 0.20919 & 0.21049 & 14603.40 & 0.16350 & 0.26031 \\
\hline ySigma[2,2] Test F & 0.26754 & 0.26557 & 0.26199 & 14210.70 & 0.20777 & 0.33139 \\
\hline sigmaMode & 0.18301 & 0.18314 & 0.18511 & 14296.90 & 0.07639 & 0.28490 \\
\hline sigmaSD & 0.13849 & 0.12181 & 0.09648 & 13128.40 & 0.02944 & 0.28913 \\
\hline Nu & 36.56547 & 27.80642 & 13.45532 & 11282.90 & 3.14193 & 95.40764 \\
\hline
\end{tabular}

\section{INTERPRETATION OF THE RESULTS}

Table 1 indicates that the baseline CA across both genders and both treatments is about 0.36 (shown as parameter b0), but there is large variation across genders and methods of treatments. For example, on average, CA for males is about 0.058 less than the baseline (shown as parameter b2[1]). To those deflections from baseline due to treatment, we also add deflections due to 
gender. For example, on average, test method shows $0.068 \mathrm{CA}$ values less than baseline (shown as parameter b1[2]).

The predicted $\mathrm{CA}$ value from the main effects alone is the sum of their deflections. For example, the additive prediction for the CA of a male is the baseline plus the main-effect deflection for gender plus the main-effect deflection for test method, b0 $+\mathrm{b} 2[1]+\mathrm{b} 1[2]$. The coefficients $\beta_{[j]}$ indicates how much the predicted value of $y$ changes when $x$ changes from neutral to category $j$. The deflections sum to zero across the categories, but the actual CA value in that cell may differ from that additive prediction, and the estimated interaction deflection is also shown in Table 1 as parameter b1b2[2,1] (which has a value of about 0.013). Thus, the predicted CA value for males treated by the test method is 0.25 , calculated by $b 0+b 2[1]+b 1[2]+b 1 b 2[2,1]$. This sum is reported in the full summary table as the parameter $\mathrm{m}[2,1]$ in Table 1 . The predicted CA values for females treated by test method is 0.34 . The predicted CA values for males treated by control method is 0.36 and the predicted CA values for females treated by control method is 0.5 .

Individual CA values vary tremendously around the predicted cell mean. The estimated standard deviation within a cell is shown in the final rows of Table 1 as parameters ySigma[,]. These estimates assume there is different standard deviation in every cell, as shown graphically by the posterior predictive distributions plotted in Figure 2. Visual inspection of the plot suggests that the assumption of heterogeneous variance is a good description of the data, because some cells have data tightly clustered while other cells have data extensively spread out. We have used a model that has different standard deviation parameters for every cell.

The estimate of interaction is more uncertain than the estimates of the main effects. This is caused by the fact that it involves four sources of uncertainty (i.e. four groups of data), unlike main effects which each involve only half of those sources of uncertainty. Interaction requires more data to estimate accurately.

\section{Conclusions}

The parameter estimates provided meaningful information about the trends in the CA data and the uncertainty in those trends. Both the compared methods showed good results in terms of CA evaluated parameters but the value of CA was higher for the women in the control group. The results of our study also demonstrate a good potential for PRFm used in the treatment of Miller's Class I 
and Class II gingival recessions and CA estimated value in women is also higher.

\section{COMPETING INTERESTS}

Authors have declared that no competing interests exist.

\section{ACKNOWLEDGMENTS}

This research is partially supported by the grant of NPD Plovdiv University, Project No: SP15-FMIIT-015.

\section{References}

[1] K.M. Albandar, A. Kingman, Gingival recession, gingival bleeding, and dental calculus in adults 30 years of age and older in the United states, 1988-1994, J Periodontol, (1999), 70:30-43.

[2] K. Anilkumar, A. Geetha, Umasudhakar et al., Platelet-rich fibrin:A novel root coverage approach, Journal of Indian Society of Periodontology (2009), 13 (1), 50-54.

[3] S. Aroca, T. Keglevich, B. Barbieri, I. Gera, D. Etienne, Clinical Evaluation of a Modified Coronally Advanced Flap Alone or in Combination With a Platelet-Rich Fibrin Membrane for the Treatment of Adjacent Multiple Gingival Recessions: A 6-Month Study, J Periodontol, (2009), Feb, 80(2):244-52.

[4] Iv. Chenchev, D. Atanasov, D. Vicheva, The treatment of gingival recessions - Our experience, Romanian Journal of Rhinology, Vol. 6, No. 22, April-June (2016), pp 85-91.

[5] Iv. Chenchev, D. Atanasov, D. Vicheva, V. Noncheva, Comparative Evaluation of the Subjective Results from the Treatment of Gingival Recessions with Connective Tissue Graft and Platelet Rich Fibrin Membrane, IOSR Journal of Dental and Medical Sciences, Vol. 15, Issue 5, Ver. 05 May (2016), pp 73-78.

[6] M. Del Cors, G. Sammartino, D.M. Dohan Ehrenfest, Re: Clinical evaluation of a modified coronally advanced flap alone or in combination with a platelet-rich fibrin membrane for the treatment of adjacent multiple gingival recessions: a 6-month study, J Periodontol (2009), 80(11):1694-7. 
[7] M. Dobreva, V. Noncheva, Iv. Chenchev, Bayesian approach for the comparison of two methods of treatment, (2015).

[8] A. Gelman, J.B Carlin, H.S Stern, D.B. Rubin, Bayesian data analysis. Third Edition, Boca Raton, Chapman and Hall-CRC, (2013).

[9] J.K. Kruschke, Doing Bayesian data analysis: A tutorial with $R$ and BUGS, Second Edition. Burlington, MA: Academic Press/Elsevier, (2011).

[10] J. Matter, G. Cimasoni, Creeping attachment after free gingival grafts, J Periodontol (1976), 47(10):574-9.

1) Faculty of Mathematics and Informatics, Plovdiv University "Paisii Hilendarski", Plovdiv, Bulgaria.

E-mail address: mimi.d.d@abv.bg

2) Faculty of Mathematics and Informatics, Plovdiv University "Paisii Hilendarski", Plovdiv, Bulgaria.

E-mail address: wesnon@uni-plovdiv.bg

3) Department of Oral Surgery, Faculty of Dental Medicine, Medical University, Plovdiv, Bulgaria .

E-mail address: ivan.chenchev@gmail.com 